Research article

\title{
Trichome diversity of the family Caryophyllaceae from Western Himalaya and their taxonomic implication
}

\author{
Satish Chandra $^{1,2} *$, D.S. Rawat ${ }^{2}$, Smriti Raj Verma $^{2}$ and Priyanka Uniyal ${ }^{3}$ \\ ${ }^{1}$ Department of Botany, Government Degree College Tiuni, Dehradun, 248199, Uttarakhand, India \\ ${ }^{2}$ Department of Biological Sciences, C.B.S.H., G.B. Pant University of Agriculture \& Technology Pantnagar- \\ 263 145, Uttarakhand, India \\ ${ }^{3}$ Department of Botany, Tehri Campus - Hemwati Nandan Bahuguna Garhwal University, Srinagar Garhwal, \\ 249001, Uttarakhand, India
}

*Corresponding Author: satishchandrasemwal07@gmail.com

[Accepted: 05 November 2019]

\begin{abstract}
Information about trichomes diversity and distribution of the family Caryophyllaceae is rare and the present work is intended to fill this knowledge lacuna. In the present work 62 taxa belonging to 19 genera were studied. For the analysis of trichomes diversity and vestiture type, dried plant specimens were rehydrated with water. The final illustrations of trichomes were made by using camera lucida. Six types of trichomes viz., Unicellular eglandular, Unicellular glandular, Multicellular uniseriate glandular, Multicellular uniseriate eglandular, Multicellular eglandular bifurcate and Multicellular multiseriate eglandular trichomes reported in the studied taxa. Diversity of trichome and their distribution does not play any significant role in the taxonomic delimitation either generic or tribal level of the family Caryophyllaceae. Although, few closely allied species can be distinguished from each other either on the basis of the presence of trichomes or vestiture patterns.
\end{abstract}

Keywords: Arenaria - Silene - Stellaria - Taxonomy -Vestiture.

[Cite as: Chandra S, Rawat DS, Verma SR \& Uniyal P (2019) Trichome diversity in the family Caryophyllaceae from Western Himalaya and their taxonomic implication. Tropical Plant Research 6(3): 397-407]

\section{INTRODUCTION}

Caryophyllaceae Juss. is known as pink family or carnation family, and ranked as $24^{\text {th }}$ largest family of Angiosperms with 93 genera and 2395 species (Thorne \& Reveal 2007). Though, number of species and genera of the family varies from different sources as: The plant list (2013) reported 88 genera with 2295 species and Mabberley (2017) reported 96 genera with 2500 species. The family is worldwide in distribution with exception in the wet tropics. Members of the family Caryophyllaceae often have distinctive swollen nodes; simple opposite leaves; dichasial cymes inflorescence or solitary flowers; flower usually actinomorphic; pentamerous with distinct clawed petals; stamens obdiplostemonous, ten or lesser; ovary superior; placentation free-central; and fruit capsule opening by teeth or valves (Bittrich 1993a, b).

On the basis of stipulate or exstipulate leaves, free or connate sepals, styles fused at base or free, the family is further subdivided by Bittrich (1993b) into three subfamilies Paronychioideae (tribe: Polycarpeae, Paronychieae and Corrigioleae), Alsinoideae (tribe: Alsineae, Pycnophylleae, Geocarpeae, Habrosieae and Sclerantheae and Caryophylloideae (tribe: Caryophylleae, Drypideae and Sileneae). Harbaugh et al. (2010) proposed a new classification for tribes of the family on the basis of monophyly and abandoned traditional three subfamily classification of family. They proposed eleven tribes namely Corrigioleae, Paronychieae, Polycarpeae, Sperguleae, Sagineae, Sclerantheae, Arenarieae, Alsineae, Eremogoneae, Caryophylleae, and Sileneae for the family.

Recently, some large genera (Arenaria, Minuartia, etc.) of the family have undergone splitting on the basis of molecular data. Dillenberger \& Kadereit (2014) divided genus Minuartia s.l. into eleven genera i.e. Cherleria, Eremogone, Facchinia, Mcnillia, Minuartia s.s., Minuartiella, Mononeuria, Pseudocherleria, Rhodalsine, Sabulina and Triplateia on the basis of monophyly. New generic delimitation for Arenaria s.l. was 
proposed by Harbaugh et al. (2010), Greenberg \& Donoghue (2011), Dillenberger \& Kadereit (2014), Sadeghian et al. (2015), and Pusalkar \& Singh (2015). In the revised circumscription Arenaria s.l. is divided into five genera i.e. Eremogone, Odontostemma, Shivparvatia, Himgiria, and Arenaria s.s.

Trichomes are epidermis originated cells found on the plant surface. There is amazing variability of trichomes in the form of variation in shape, size and structure. For the delimitation and differentiation of certain taxa variability of trichomes is used (Werker 2000). Information about trichomes diversity and distribution of the family is little known. Metcalfe \& Chalk (1950) studied trichomes diversity in some species and genera of the family. Bittrich (1993b) compiled morphological characteristics of the family and also focused on trichome diversity of some species. Some other workers Solereder \& Scott (1986), Cowie (1994), Shamsabad et al. (2013), Selvi et al. (2014), Bozchaloyi \& Keshavarz (2014) tried to document trichome diversity of the family but their study either focused on several genera or particular subfamily. Thus, present work intends to illustrate trichomes diversity and distribution in the family Caryophyllaceae and evaluate the relevance of this information for the family's taxonomy.

\section{MATERIALS AND METHODS}

\section{Plant material}

Trichome diversity and distribution were studied in 62 taxa including 58 species and four varieties belonging to 19 genera during present work. Plant specimens of 46 taxa were collected from the Western Himalaya, India during the year July 2012 to 2016 and specimens deposited in the herbarium of Govind Ballabh Pant University of Agriculture and Technology Pantnagar, Uttarakhand, India (GBPUH). Most of the specimens were collected from Uttarakhand Himalaya. Remaining taxa studied from specimens housed in herbaria of Botanical Survey of India Northern Circle Dehradun (BSD), Hemvati Nandan Bahuguna Garhwal University Srinagar Garhwal (GUH), Forest Research Institute Dehradun (DD) and Kumaon University Nainital (KUH).

\section{Light microscopy}

For the analysis of trichomes diversity and vestiture type, dried plant specimens were rehydrated with water. Trichomes from stem were obtained through scraping. Other plant parts as leaves, bracts and floral parts were observed under the light microscope and freehand horizontal and longitudinal sections were made to study trichomes morphology. The final illustrations of trichomes were made by using camera lucida. About 5-6 specimens for each taxa and 3-5 plant parts for each specimens were studied to assess the trichome diversity and distribution.

\section{Trichomes classification}

Payne (1978), Prabhakar \& Leelavathi (1989), Krak \& Mraz (2008) and de Andrade et al. (2014) were followed for classification, identification and characterization of trichomes in the present work. The vestiture types were determined following Lawrence (1951) and Simpson (2010).

\section{RESULTS}

Trichomes diversity

Six types of trichomes were recorded in the studied taxa; (1) Unicellular eglandular trichomes, (2) Unicellular glandular trichomes, (3) Multicellular uniseriate glandular trichomes, (4) Multicellular uniseriate eglandular trichomes (5) Multicellular eglandular bifurcate trichomes and (6) Multicellular multiseriate eglandular trichomes (Fig. 1). Apart from above mentioned categories stellate trichomes are also reported in Stellaria vestita Kurz, Cerastium mollissimum Poir., Polycarpon loeflingiae (Wall. ex Wight \& Arn.) Benth. \& Hook.f. (Solereder \& Scott 1986, Majumdar 1993). However, these species were not included in the present work.

On the basis of trichomes types, all the studied taxa (62 taxa) can be categorized into five groups. Stellaria depressa Em. Schmid, Spergula fallax (Lowe) E.H. Krause and Cerastium davuricum Fischer were completely glabrous and constitute the first group. Second group possess unicellular trichomes and represented by i.e. Drymaria cordata (L.) Willd. ex Schult. and Herniaria cachemiriana J. Gay. Drymaria cordata possess both unicellular eglandular and capitate unicellular glandular trichomes, while Herniaria cachemiriana possess unicellular hairs on all plant parts except ovary. In the ovary of Herniaria cachemiriana multiseriate multicellular eglandular trichomes were found.

The third group of the plants contains strictly eglandular trichomes. This group further divided into two subgroups i.e. bifurcate eglnadular trichomes and uniseriate multicellular eglandular trichome. Polycarpon prostratum (Forssk.) Asch. \& Schweinf. possesses eglandular bifurcated trichomes and also possesses simple 
multicellular uniseriate eglandular trichomes. The taxa i.e. Silene baccifera (L.) Roth, S. caespitella F. Williams, S. kumaonensis F. Williams, S. moorcroftiana Wallich ex Benth., S. nepalensis Majumdar, S. vulgaris (Moench) Garcke, Stellaria decumbens var. decumbens Edgew., S. decumbens var. polyantha Edgew. \& Hook.f., S. congestiflora H. Hara, S. himalayensis Majumdar, S. patens D.Don, S. semivestita Edgew., S. umbellata Turcz., S. uliginosa Murray, Thylacospermum caespitosum(Cambess.) Schischk., Cherleria sp., Drymaria villosa Cham. \& Schlecht., Dianthus angulatus Royle ex Benth., D. barbatus L., D. chinensis L., Arenaria thangoensis W.W. Sm., Stellaria depauperata Edgew., Arenaria bhutanica Majumdar \& Babu, Eremogone ferruginea (Duthie ex F.Williams) Pusalkar \& D.K. Singh, E. curvifolia Kar. \& Kir., E. kumaonensis, Shivparvatia ciliolata var. ciliolata (Edgew.) Pusalkar \& D.K. Singh, S. ciliolata var. pendula (Duthie ex F. Williams) Pusalkar \& D.K. Singh, Gypsophila cerastioides D.Don, Sagina saginoides (L.) Karsten of this group possess only simple uniseriate multicellular eglandular trichomes. Only eglandular trichomes present on at least one plant part of this group.

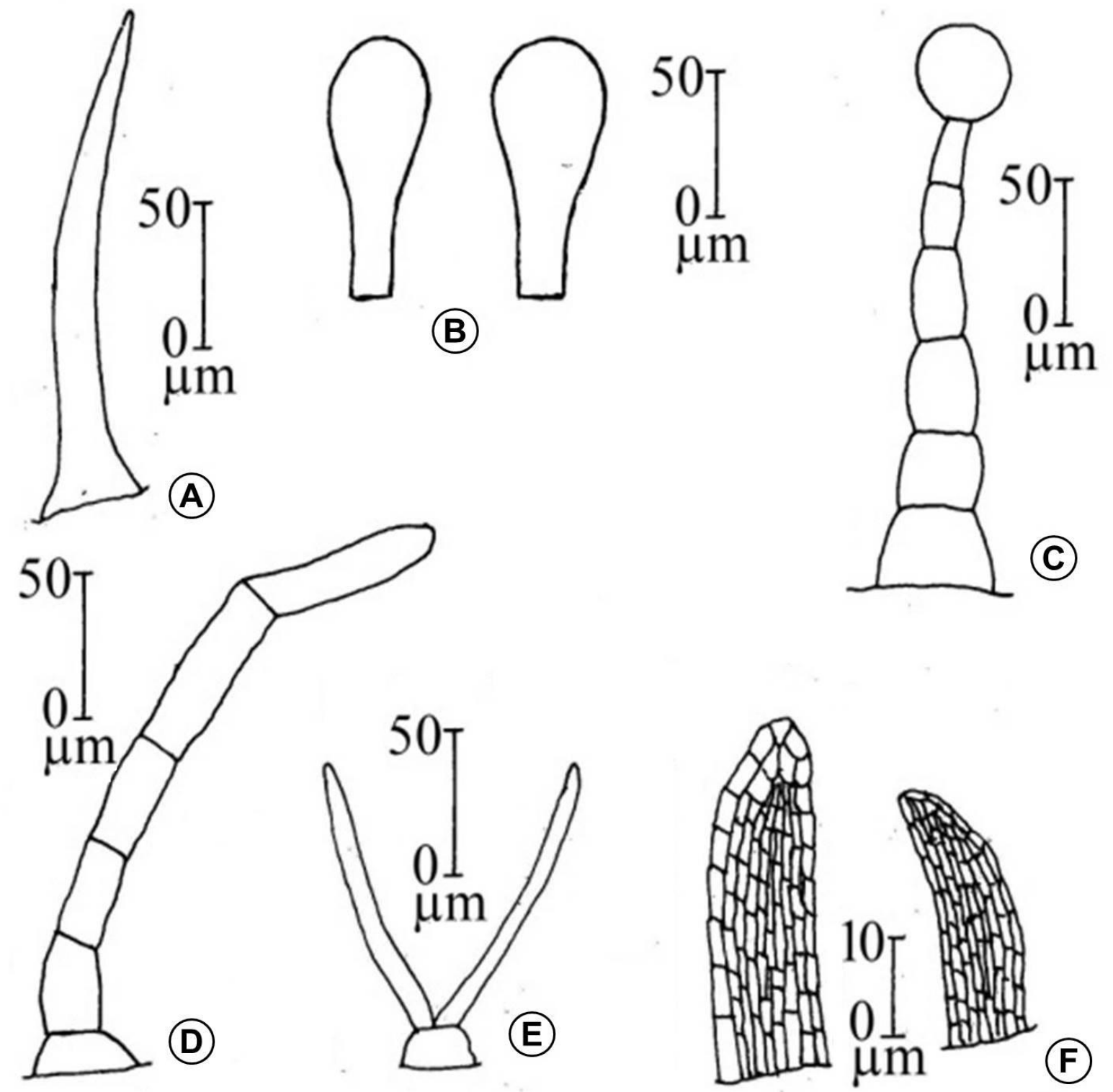

Figure 1. Different types of trichomes in family Caryophyllaceae: A, Unicelluar eglandular trichome (Herniaria cachemiriana); B, Unicelluar glandular trichome (Drymaria cordata); C, Mulicellular uniseriate glandular trichome (Shivparvatia glanduligera); D, Mulicellular uniseriate eglandular trichome (Arenaria bhutanica); E, Mulicellular eglandular bifurcate trichome (Polycarpon prostratum); F, Multicellular multiseriate eglandular trichome (Herniaria cachemiriana).

The forth group of the plants bore only uniseriate multicellular glandular trichomes and represented by Polycarpaea corymbosa (L.) Lam. and Spergularia rubra (L.) J. \& K. Presl. The fifth group include Silene falconeriana Royle ex Benth., S. conoidea L., S. gangotriana Pusalkar, D.K.Singh \& Lakshmin., S. indica var. indica Roxb. ex Otth, S. indica var. edgeworthii (Bocquet) Y.J. Nasir., S. incurvifolia Kar. \& Kir., S. viscosa (L.) Pers., Stellaria aquatica (L.) Scop., S. media (L.) Villars, S. monosperma var. monosperma D.Don, S. www.tropicalplantresearch.com 
monosperma var. paniculata (Edgew.) Majumdar, S. webbiana (Benth. ex G. Don) Edgew. \& Hook. f., Cerastium cerastioides (L.) Britton, C. fontanum Baumg., Odontostemma glandulosum Benth. ex D.Don, Eremogone festucoides (Benth.) Pusalkar \& D.K. Singh, Shivparvatia glanduligera (Edgew.) Pusalkar \& D.K. Singh, Arenaria neelgherrensis Wight \& Arn., A. serpylifolia L., Lepyrodiclis holosteoides (C. A. Meyer) Fenzl ex Fischer \& C. A. Meyer and Sagina apetala Ard., which possessed both unseriate multicellular glandular and eglandular trichomes at least at some plant parts.

Presence of trichomes in different plant parts

Trichomes in all plants are not uniform in length and distribution. Hence, the presence of trichomes in different plant parts and different types of vestiture can also be used for categorization of taxa. The presence of trichomes in different plant parts of studied taxa is shown in table 1. Stellaria depressa, Spergula fallax and Cerastium davuricum were completely glabrous nature. Dianthus barbatus L., D. chinensis and Silene vulgaris (Moench) Garcke had trichomes only at calyx teeth apex, otherwise these were glabrous plants. In Thylacospermum caespitosum trichomes were found only at abaxial surface of leaf, while in Stellaria uliginosa Murray, S. umbellata trichomes were found at abaxial surface of leaf toward leaf base and leaf base of upper few leaf pairs respectively. In Polycarpaea corymbosa trichomes found only upper parts of stem and pedicel. Among all species, only Herniaria cachemiriana had trichomes on the ovary surface, while in all other taxa ovary were glabrous.

Table 1. Presence of trichomes on different plant parts of the studied taxa.

\section{S.N. Name of Taxa}

Arenaria bhutanica Majumdar \& Babu

Arenaria neelgherrensis Wight \& Arn.

Arenaria orbiculata Royle ex Edgew. \& Hook.f.

$4 \quad$ Arenaria serpylifolia $\mathrm{L}$.

5 Arenaria thangoensis W.W. Sm.

6 Cerastium cerastoides (L.) Britton

7 Cerastium davuricum Fischer

8 Cerastium fontanum Baumg.

9 Cherleria sp.

10 Dianthus angulatus Royle ex Benth.

11 Dianthus barbatus L.

12 Dianthus chinensis L.

13 Drymaria cordata (L.) Willd. ex Schult.

14 Drymaria villosa Cham. \& Schlecht.

15 Eremogone curvifolia (Majumdar)

Pusalkar \& D.K. Singh

16 Eremogone festucoides (Benth.)

Pusalkar \& D.K. Singh

17 Eremogone ferruginea (Duthie ex F.Williams) Pusalkar \& D.K. Singh

18 Eremogone kumaonensis (Maxim.) Pusalkar \& D.K. Singh

19 Gypsophila cerastioides D.Don

20 Herniaria cachemiriana J. Gay

21 Lepyrodiclis holosteoides (C. A. Meyer) Fenzl ex Fischer \& C. A. Meyer

22 Odontostemma glandulosum Benth. ex D.Don

23 Polycarpaea corymbosa (L.) Lam.

24 Polycarpon prostratum (Forssk.) Asch. \& Schweinf.

25 Sabulina kashmirica (Edgew.) Dillenb. \& Kadereit

26 Sagina apetala Ard.

27 Sagina saginoides (L.) Karsten

28 Shivparvatia ciliolata (Edgew.) Pusalkar \& D.K. Singh var. ciliolate (Edgew.) Pusalkar \& D.K. Singh

\section{Presence of Trichomes on different plant parts}

\begin{tabular}{|c|c|c|c|c|c|c|c|c|c|c|c|c|}
\hline St & Lad & Lab & Lm & Lb & B & $\mathbf{P}$ & $\mathbf{C b}$ & Ca & $\mathbf{P t}$ & $\mathbf{S}$ & O & Sti \\
\hline$\checkmark$ & $x$ & $x$ & $x$ & $\checkmark$ & $x$ & $\checkmark$ & $x$ & $x$ & $x$ & $x$ & $x$ & $x$ \\
\hline$\checkmark$ & $x$ & $x$ & $\checkmark$ & $\checkmark$ & $x$ & $\checkmark$ & $x$ & $x$ & $x$ & $x$ & $x$ & $x$ \\
\hline$\checkmark$ & $x$ & $\checkmark$ & $x$ & $x$ & $x$ & $\checkmark$ & $x$ & $x$ & $x$ & $x$ & $x$ & $x$ \\
\hline$\checkmark$ & $\checkmark$ & $\checkmark$ & $\checkmark$ & $\checkmark$ & $\checkmark$ & $\checkmark$ & $\checkmark$ & $\checkmark$ & $x$ & $x$ & $x$ & $x$ \\
\hline$\checkmark$ & $\checkmark$ & $\checkmark$ & $\checkmark$ & $\checkmark$ & $\checkmark$ & $\checkmark$ & $\checkmark$ & $\checkmark$ & $x$ & $x$ & $x$ & $x$ \\
\hline$\checkmark$ & $x$ & $x$ & $x$ & $\checkmark$ & $x$ & $\checkmark$ & $\checkmark$ & $\checkmark$ & $x$ & $x$ & $x$ & $x$ \\
\hline$x$ & $x$ & $x$ & $x$ & $x$ & $x$ & $x$ & $x$ & $x$ & $x$ & $x$ & $x$ & $x$ \\
\hline$\checkmark$ & $\checkmark$ & $\checkmark$ & $\checkmark$ & $\checkmark$ & $\checkmark$ & $\checkmark$ & $\checkmark$ & $\checkmark$ & $x$ & $x$ & $x$ & $x$ \\
\hline$\checkmark$ & $x$ & $x$ & $\checkmark$ & $\checkmark$ & $\checkmark$ & $\checkmark$ & $\checkmark$ & $\checkmark$ & $x$ & $x$ & $x$ & $x$ \\
\hline$\checkmark$ & $x$ & $x$ & $\checkmark$ & $\checkmark$ & $x$ & $\checkmark$ & $\checkmark$ & $\checkmark$ & $x$ & $x$ & $x$ & $x$ \\
\hline$x$ & $x$ & $x$ & $x$ & $x$ & $x$ & $x$ & $x$ & $\checkmark$ & $x$ & $x$ & $x$ & $x$ \\
\hline$x$ & $x$ & $x$ & $x$ & $x$ & $x$ & $x$ & $x$ & $\checkmark$ & $x$ & $x$ & $x$ & $x$ \\
\hline$\checkmark$ & $x$ & $x$ & $x$ & $\checkmark$ & $\checkmark$ & $\checkmark$ & $\checkmark$ & $\checkmark$ & $x$ & $x$ & $x$ & $x$ \\
\hline$\checkmark$ & $\checkmark$ & $\checkmark$ & $\checkmark$ & $\checkmark$ & $\checkmark$ & $\checkmark$ & $\checkmark$ & $\checkmark$ & $x$ & $x$ & $x$ & $x$ \\
\hline$\checkmark$ & $x$ & $x$ & $x$ & $\checkmark$ & $x$ & $\checkmark$ & $\checkmark$ & $x$ & $x$ & $x$ & $x$ & $x$ \\
\hline$\checkmark$ & $\checkmark$ & $x$ & $x$ & $\checkmark$ & $\checkmark$ & $\checkmark$ & $\checkmark$ & $\checkmark$ & $x$ & $x$ & $x$ & $x$ \\
\hline$\checkmark$ & $x$ & $x$ & $x$ & $\checkmark$ & $x$ & $\checkmark$ & $\checkmark$ & $x$ & $x$ & $x$ & $x$ & $x$ \\
\hline$x$ & $x$ & $x$ & $x$ & $\checkmark$ & $\checkmark$ & $\checkmark$ & $\checkmark$ & $x$ & $x$ & $x$ & $x$ & $x$ \\
\hline$\checkmark$ & $\checkmark$ & $\checkmark$ & $\checkmark$ & $\checkmark$ & $\checkmark$ & $\checkmark$ & $\checkmark$ & $\checkmark$ & $x$ & $x$ & $x$ & $x$ \\
\hline$\checkmark$ & $\checkmark$ & $\checkmark$ & $\checkmark$ & $\checkmark$ & $x$ & $x$ & $\checkmark$ & $\checkmark$ & $x$ & $x$ & $\checkmark$ & $\checkmark$ \\
\hline$\checkmark$ & $\checkmark$ & $\checkmark$ & $\checkmark$ & $\checkmark$ & $\checkmark$ & $\checkmark$ & $\checkmark$ & $\checkmark$ & $x$ & $x$ & $x$ & $x$ \\
\hline$\checkmark$ & $\checkmark$ & $\checkmark$ & $\checkmark$ & $\checkmark$ & $\checkmark$ & $\checkmark$ & $\checkmark$ & $\checkmark$ & $x$ & $x$ & $x$ & $x$ \\
\hline$\checkmark$ & $\checkmark$ & $x$ & $x$ & $x$ & $\checkmark$ & $x$ & $x$ & $x$ & $x$ & $x$ & $x$ & $x$ \\
\hline$\checkmark$ & $\checkmark$ & $\checkmark$ & $\checkmark$ & $\checkmark$ & $\checkmark$ & $\checkmark$ & $\checkmark$ & $\checkmark$ & $x$ & $x$ & $x$ & $\checkmark$ \\
\hline$x$ & $x$ & $x$ & $x$ & $x$ & $x$ & $x$ & $x$ & $x$ & $x$ & $x$ & $x$ & $x$ \\
\hline$\checkmark$ & $x$ & $x$ & $\checkmark$ & $x$ & $x$ & $\checkmark$ & $x$ & $x$ & $x$ & $x$ & $x$ & $x$ \\
\hline$\checkmark$ & $x$ & $x$ & $\checkmark$ & $x$ & $x$ & $\checkmark$ & $x$ & $x$ & $x$ & $x$ & $x$ & $x$ \\
\hline$\checkmark$ & $x$ & $x$ & $\checkmark$ & $\checkmark$ & $\checkmark$ & $\checkmark$ & $\checkmark$ & $\checkmark$ & $x$ & $x$ & $x$ & $x$ \\
\hline
\end{tabular}


29 Shivparvatia ciliolata var. pendula (Duthie ex F. Williams) Pusalkar \& D.K. Singh

30 Shivparvatia glanduligera (Edgew.) Pusalkar \& D.K. Singh

31 Silene baccifera (L.) Roth

32 Silene caespitella $\mathrm{F}$. Williams

33 Silene conoidea $\mathrm{L}$.

34 Silene falconeriana Royle ex Benth.

35 Silene gangotriana Pusalkar, D.K.Singh \& Lakshmin.

36 Silene incurvifolia Kar. \& Kir.

37 Silene indica var. edgeworthii (Bocquet) Y.J. Nasir

38 Silene indica Roxb. ex Otth var. indica Roxb. ex Otth

39 Silene kumaonensis F. Williams

40 Silene moorcroftiana Wallich ex Benth.

41 Silene nepalensis Majumdar

42 Silene songarica (Fisch., C.A. Mey. \& Ave-Lall.) Bocq

43 Silene viscosa (L.) Pers.

44 Silene vulgaris (Moench) Garcke

45 Spergula fallax (Lowe) E.H. Krause

46 Spergularia rubra (L.) J. \& K. Presl.

47 Stellaria aquatica (L.) Scop.

48 Stellaria congestiflora H. Hara

49 Stellaria decumbens Edgew. var. decumbens Edgew.

50 Stellaria decumbens Edgew. var. polyantha Edgew.\& Hook.f.

51 Stellaria depauperata Edgew.

52 Stellaria depressa Em. Schmid

53 Stellaria himalayensis Majumdar

54 Stellaria media (L.) Villars

55 Stellaria monosperma D.Don var. monosperma D.Don

56 Stellaria monosperma D.Don var. paniculata (Edgew.) Majumdar

57 Stellaria patens D.Don

58 Stellaria semivestita Edgew.

59 Stellaria umbellata Turcz.

60 Stellaria uliginosa Murray

61 Stellaria webbiana (Benth. ex G. Don) Edgew. \& Hook. f.

62 Thylacospermum caespitosum (Cambess.) Schischk.

Note: $\mathrm{St}=\mathrm{Stem} ; \mathrm{Lad}=$ Leaf adaxial surface; $\mathrm{Lab}=\mathrm{Leaf}$ abaxial surface; $\mathrm{Lm}=$ Leaf margin; $\mathrm{Lb}=$ Leaf base; $\mathrm{B}=\mathrm{Bract} ; \mathrm{P}=\mathrm{Pedicel} ; \mathrm{Cb}=$ Calyx base; $\mathrm{Ca}=$ Calyx apex; $\mathrm{Pt}=$ Petal; $\mathrm{S}=\mathrm{Stamen} ; \mathrm{O}=$ Ovary; $\mathrm{Sti}=$ Stipule.

\section{Vestiture Type}

Different types of vestiture depict relative length and pattern of trichome and hence can be used for further categorization of taxa. Different arrangements of trichomes on the stem surface were studied. In the plants under study, eight different types of vestitures were recorded viz. (1) Pubescent- more or less straight, short, soft, somewhat scattered, slender trichomes, (2) Puberulent- minutely pubescent with very short scattered trichomes, (3) Tomentose- plant parts covered with very dense interwoven trichomes, (4) Villous- long, soft, highly twisted trichomes, (5) Scabrous- rough trichomes, as of sandpaper, (6.) Hirsute- long stiff trichomes, (7) Strigose- dense coarse, bent and flat trichomes often with bulbous base, and (8) Strigulose- minutely strigose.

Stellaria monosperma var. paniculata and S. monosperma var. monosperma, S. media, S. aquatica, Shivparvatia ciliolata var. ciliolata, and Shivparvatia ciliolata var. pendula had tomentose vestiture. But, these were further differentiated on the basis of single line of trichomes, two lines of trichomes or diffused trichomes 
throughout the stem. Stellaria monosperma var. paniculata and S. monosperma var. paniculata both possess trichomes in one line; Stellaria media has two lines of trichomes or sometimes diffused, Shivparvatia ciliolata var. ciliolata, Shivparvatia ciliolata var. pendula has two lines of trichomes. Arenaria orbiculata Royle ex Edgew. \& Hook.f. and Stellaria aquatica both possess two lines of trichomes, but in Arenaria orbiculata vestiture is scabrous, while in Stellaria aquatica vestiture is tomentose. Shivparvatia glanduligera, Silene conoidea, S. falconeriana, S. nepalensis, Stellaria depauperata and Arenaria bhutanica had pubescent vestiture. Stellaria depauperata and Arenaria bhutanica are distinguished due to presence of only one line of trichomes in Arenaria bhutanica and both one and two lines of trichomes are present in Stellaria depauperata. Shivparvatia glanduligera possess trichomes either in two lines or in diffused manner and hence separated from the rest of taxa. The species Silene conoidea, S. falconeriana, S. nepalensis and rest of the species possess trichomes in diffused manner. The presence of different types of vestiture in studied taxa is summarized in table 2 . In figure 2 , representatives of aforementioned eight categories are depicted.

Table 2. Trichome types and vestiture of studied plant taxa of the family Caryophyllaceae.

\begin{tabular}{|c|c|c|c|}
\hline S.N. Name of Taxa & $\begin{array}{l}\text { Specimen } \\
\text { Number }\end{array}$ & $\begin{array}{l}\text { Trichome } \\
\text { Type* }\end{array}$ & $\begin{array}{l}\text { Vestiture } \\
\text { Type }\end{array}$ \\
\hline 1. Arenaria bhutanica Majumdar \& Babu & GBPUH823 & $\mathrm{E}$ & Pubescent \\
\hline 2. Arenaria neelgherrensis Wight \& Arn. & GBPUH810 & $\mathrm{E}, \mathrm{G}$ & Scabrous \\
\hline 3. Arenaria orbiculata Royle ex Edgew. \& Hook.f. & GBPUH813 & E & Scabrous \\
\hline 4. Arenaria serpylifolia $\mathrm{L}$. & GBPUH816 & $\mathrm{E}, \mathrm{G}$ & Scabrous \\
\hline 5. Arenaria thangoensis W.W. Sm. & GBPUH826 & $\mathrm{E}$ & Pubescent \\
\hline 6. Cerastium cerastoides (L.) Britton & BSD103333 & $\mathrm{E}, \mathrm{G}$ & Tomentose \\
\hline 7. Cerastium davuricum Fischer & BSD89568 & Glabrous & Glabrous \\
\hline 8. Cerastium fontanum Baumg. & GBPUH830 & $\mathrm{E}, \mathrm{G}$ & Hirsute \\
\hline 9. Cherleria sp. & GBPUH609 & $\mathrm{E}$ & Puberulent \\
\hline 10. Dianthus angulatus Royle ex Benth. & BSD101853 & $\mathrm{E}$ & Puberulent \\
\hline 11. Dianthus barbatus L. & GBPUH759 & $\mathrm{E}$ & Glabrous \\
\hline 12. Dianthus chinensis L. & GBPUH760 & $\mathrm{E}$ & Glabrous \\
\hline 13. Drymaria cordata (L.) Willd. ex Schult. & GBPUH762 & $\mathrm{CG}, \mathrm{UE}$ & Scabrous \\
\hline 14. Drymaria villosa Cham. \& Schlecht. & GBPUH745 & $\mathrm{E}$ & Villous \\
\hline $\begin{array}{l}\text { 15. Eremogone curvifolia (Majumdar) Pusalkar \& D.K. } \\
\text { Singh }\end{array}$ & GBPUH819 & $\mathrm{E}$ & Puberulent \\
\hline 16. Eremogone festucoides (Benth.) Pusalkar \& D.K. Singh & GBPUH820 & $\mathrm{E}, \mathrm{G}$ & Tomentose \\
\hline $\begin{array}{l}\text { 17. Eremogone ferruginea (Duthie ex F.Williams) Pusalkar } \\
\text { \& D.K. Singh }\end{array}$ & DD5388 & $\mathrm{E}$ & Strigose \\
\hline $\begin{array}{l}\text { 18. Eremogone kumaonensis (Maxim.) Pusalkar \& D.K. } \\
\text { Singh }\end{array}$ & GBPUH821 & $\mathrm{E}$ & Tomentose \\
\hline 19. Gypsophila cerastioides D.Don & GBPUH757 & $\mathrm{E}$ & Tomentose \\
\hline 20. Herniaria cachemiriana J. Gay & BSD103341 & MSE, UE & Hirsute \\
\hline $\begin{array}{l}\text { 21. Lepyrodiclis holosteoides (C. A. Meyer) Fenzl ex } \\
\text { Fischer \& C. A. Meyer }\end{array}$ & BSD7340 & $\mathrm{E}, \mathrm{G}$ & Tomentose \\
\hline 22. Odontostemma glandulosum Benth. ex D.Don & GBPUH825 & $\mathrm{E}, \mathrm{G}$ & Hirsute \\
\hline 23. Polycarpaea corymbosa (L.) Lam. & BSD67856 & G & Tomentose \\
\hline 24. Polycarpon prostratum (Forssk.) Asch. \& Schweinf. & BSD32008 & $\mathrm{BE}, \mathrm{E}$ & Tomentose \\
\hline 25. Sabulina kashmirica (Edgew.) Dillenb. \& Kadereit & GBPUH806 & Glabrous & Glabrous \\
\hline 26. Sagina apetala Ard. & GUH12807 & $\mathrm{E}, \mathrm{G}$ & Puberulent \\
\hline 27. Sagina saginoides (L.) Karsten & GBPUH766 & $\mathrm{E}$ & Puberulent \\
\hline $\begin{array}{l}\text { 28. Shivparvatia ciliolata (Edgew.) Pusalkar \& D.K. Singh } \\
\text { var. ciliolata }\end{array}$ & GBPUH807 & $\mathrm{E}$ & Tomentose \\
\hline $\begin{array}{l}\text { 29. Shivparvatia ciliolata var pendula (Duthie ex F. } \\
\text { Williams) Pusalkar \& D.K. Singh }\end{array}$ & GBPUH808 & $\mathrm{E}$ & Tomentose \\
\hline $\begin{array}{l}\text { 30. Shivparvatia glanduligera (Edgew.) Pusalkar \& D.K. } \\
\text { Singh }\end{array}$ & GBPUH809 & $\mathrm{E}, \mathrm{G}$ & Pubescent \\
\hline 31. Silene baccifera $($ L.) Roth & GBPUH770 & $\mathrm{E}$ & Strigose \\
\hline 32. Silene caespitella $\mathrm{F}$. Williams & BSD1102 & $\mathrm{E}$ & Strigose \\
\hline 33. Silene conoidea $\mathrm{L}$. & GBPUH772 & $\mathrm{E}, \mathrm{G}$ & Pubescent \\
\hline 34. Silene falconeriana Royle ex Benth. & GBPUH773 & $\mathrm{E}, \mathrm{G}$ & Pubescent \\
\hline 35. Silene gangotriana Pusalkar, D.K.Singh \& Lakshmin. & GBPUH774 & $\mathrm{E}, \mathrm{G}$ & Tomentose \\
\hline 36. Silene incurvifolia Kar. \& Kir. & GBPUH777 & $\mathrm{E}, \mathrm{G}$ & Strigulose \\
\hline 37. Silene indica var. edgeworthii (Bocquet) Y.J. Nasir. & GBPUH780 & $\mathrm{E}, \mathrm{G}$ & Strigulose \\
\hline 38. Silene indica Roxb. ex Otth var. indica & GBPUH779 & $\mathrm{E}, \mathrm{G}$ & Tomentose \\
\hline
\end{tabular}




\begin{tabular}{|c|c|c|c|}
\hline 39. Silene kumaonensis F. Williams & BSD93913 & $\mathrm{E}$ & Tomentose \\
\hline 40. Silene moorcroftiana Wallich ex Benth. & BSD56258 & $\mathrm{E}$ & Strigose \\
\hline 41. Silene nepalensis Majumdar & GBPUH781 & $\mathrm{E}$ & Pubescent \\
\hline 42. Silene songarica (Fisch., C.A. Mey. \& Ave-Lall.) Bocq & BSD103901 & E & Tomentose \\
\hline 43. Silene viscosa (L.) Pers. & BSD102127 & $\mathrm{E}, \mathrm{G}$ & Tomentose \\
\hline 44. Silene vulgaris (Moench) Garcke & GBPUH785 & E & \\
\hline 45. Spergula fallax (Lowe) E.H. Krause & GBPUH765 & Glabrous & Glabrous \\
\hline 46. Spergularia rubra (L.) J. \& K. Presl. & BSD13009 & G & Puberulent \\
\hline 47. Stellaria aquatica (L.) Scop. & BSD14901 & $\mathrm{E}, \mathrm{G}$ & Tomentose \\
\hline 48. Stellaria congestiflora $\mathrm{H}$ & GBPUH704 & E & Villous \\
\hline 49. Stellaria decumbens Edgew. var. decumbens Edgew. & GBPUH787a & $\mathrm{E}$ & Strigulose \\
\hline $\begin{array}{l}\text { 50. Stellaria decumbens Edgew. var. polyantha Edgew.\& } \\
\text { Hook.f. }\end{array}$ & GBPUH787b & $\mathrm{E}$ & Strigulose \\
\hline 51. Stellaria depauperata Edgew. & GBPUH749 & $\mathrm{E}$ & Pubescent \\
\hline 52. Stellaria depressa Em. Schmid & KUH1788 & Glabrous & Glabrous \\
\hline 53. Stellaria himalaye & & E & Villous \\
\hline 54. Stellaria media (L.) Villars & GBPUH784 & $\mathrm{E}, \mathrm{G}$ & Tomentose \\
\hline 55. Stellaria monosperma D.Don var. monosperma & GBPUH788 & $\mathrm{E}, \mathrm{G}$ & Tomentose \\
\hline $\begin{array}{l}\text { 56. Stellaria monosperma D.Don var. paniculata (Edgew.) } \\
\text { Majumdar }\end{array}$ & GBPUH788 & $\mathrm{E}, \mathrm{G}$ & Tomentose \\
\hline 57. Stellaria patens D.Don & JH791 & $\mathrm{E}$ & Villous \\
\hline 58. Stellaria semivestita Edgew. & GBPUH796 & $\mathrm{E}$ & Villous \\
\hline 59. Stellaria umbellata Turcz. & GBPUH802 & $\mathrm{E}$ & Villous \\
\hline 60. Stellaria uliginosa Murray & GBPUH803 & E & Glabrous \\
\hline $\begin{array}{l}\text { 61. Stellaria webbiana (Benth. ex G. Don) Edgew. \& Hook. } \\
\text { f. }\end{array}$ & GBPUH805 & $\mathrm{E}, \mathrm{G}$ & Strigulose \\
\hline 62. Thylacospermum caespitosum (Cambess.) Schischk. & BSD101818 & $\mathrm{E}$ & Glabrous \\
\hline
\end{tabular}

Note: $\mathrm{BE}=$ Bifurcate Eglandular; $\mathrm{CG}=$ Capitate Glandular; $\mathrm{E}=$ Uniseriate Multicellular Eglandular; $\mathrm{G}=$ Uniseriate Multicellular Glandular; MSE= Multiseriate Eglandular, UE= Unicellular Eglandular.
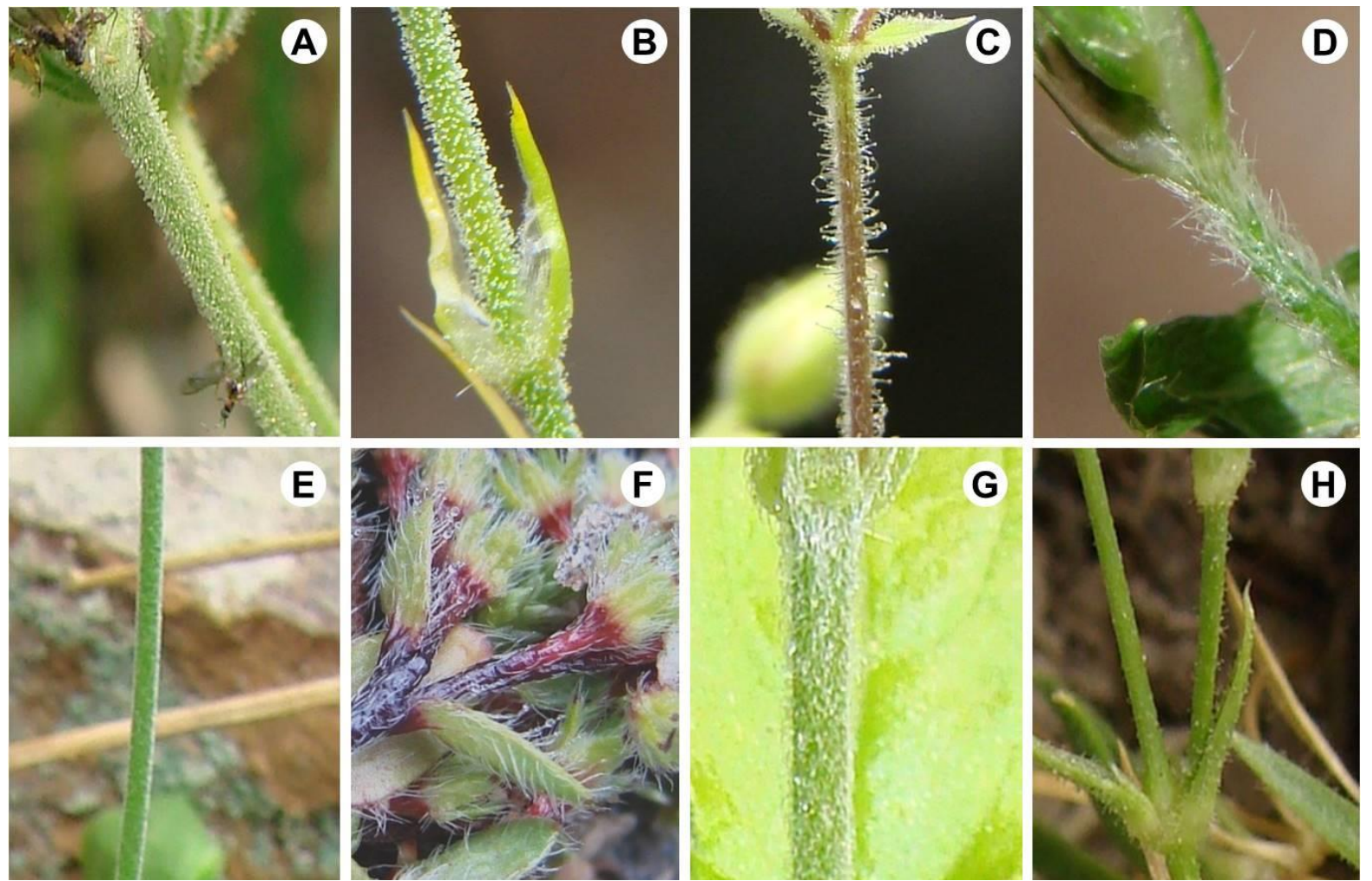

Figure 2. Different types of vestiture: A, Pubescent (Silene conoidea); B, Puberulent (Eremogone curvifolia); C, Tomentose (Stellaria monosperma var. monosperma); D, Villous (Stellaria patens); E, Scabrous (Drymaria cordata); F, Hirsute (Herniaria cachemiriana); G, Strigose (Silene baccifera); H, Strigulose (Stellaria webbiana).

\section{DISCUSSION}

Some of the investigated species had peculiar trichome morphology which can be used to distinguish them from the rest of the allied species. Moreover, trichome morphology and distribution do not play any significant 
role in separation of genera. Herniaria cachemiriana has long unicellular stiff eglandular trichomes on different plant parts and multiseriate multicellular egladular trichomes on the ovary. Metcalfe \& Chalk (1950) also mentioned unicellular tricomes in Herniaria, Paronychia, Anychia and Siphonychia genus. Multiseriate trichomes are also reported from Achyronichia spp., Cerastium spp., Pollichia spp., Polycarpon spp., Polycarpaea spp., Polytepalum spp., Krauseola spp. and Stipulicida spp. (Bittrich 1993b). But the species of Cerastium, Polycarpon and Polycarpaea studied in the present work does not contain multiseriate trichomes. Drymaria cordata is characterized by presence of unicellular capitate glandular trichomes on stem, pedicel and sepal abaxial surface. Metcalfe \& Chalk (1950) and Keshavarzi \& Bozchaloyi (2014) also reported similar type of unicellular sessile capitate glandular trichomes in Viscaria spp. and Silene propinqua Schischk. Polycarpon prostratum possesses multicellular eglandular bifurcate trichomes and same type of trichomes also known in Pollichia campestris Aiton (Solereder \& Scott 1986). Solereder \& Scott (1986) reported eglandular trichomes containing two or more arms in Cerastium dicrotrichum Fenzl ex Rohrb., Polycarpaea spp. including $P$. teneriffae Lam. and Stipulicida spp.

Uniseriate multicellular eglandular or glandular trichomes are present in the Silene, Stellaria, Arenaria, Odontostemma, Cherleria, Dianthus, Drymaria, Eremogone, Gypsophila, Lepyrodiclis, Polycarpaea, Sagina, Shivparvatia, Spergularia and Thylacospermum possess. Similar uniseriate glandular or eglandular trichomes are also reported from Acanthophyllum spp., Stellaria spp., Agrostemma spp. (Shamsabad et al. 2013, Keshavarzi \& Bozchaloyi 2014, Selvi et al. 2014). Keshavarzi \& Bozchaloyi (2014) examined some taxa of subfamily Alsinoideae and found simple eglandular uniseriate trichomes in most of the taxa. Genera of subfamily Alsinoideae included in the present study are Stellaria, Arenaria, Odontostemma, Cherleria, Eremogone, Lepyrodiclis, Sagina, Shivparvatia and Thylacospermum which show similar morphology of trichomes as reported by Keshavarzi \& Bozchaloyi (2014) in the subfamily.

Unseriate multicellular eglandular and glandular trichomes present in all species of Silene understudy. Yildiz \& Minareci (2008), Kilic (2009), Sahreen et al. (2010), Khan et al. (2013), Bagci \& Bicer (2015) have also reported similar type of trichomes in different Silene species. All Stellaria species understudy possess uniseriate multicellular glandular or eglandular trichomes which correspond well with the results of Bozchaloyi \& Keshavarzi (2014) who reported similar type of trichomes in different Stellaria species. During the present study uniseriate multicellular glandular and eglandular trichomes were found in Cerastium fontanum and $C$. cerastioides. Arcus et al. (2012) have also reported similar trichomes in Cerastium bulgaricum Uechtr., $C$. tomentosum L., C. brachypetalum Desp. ex Pers. and C. glomeratum Thuill. Gypsophila cerastioides possess only uniseriate multicellular eglandular trichomes but uniseriate multicellular glandular trichomes also were found in G. lepidioides Boiss. (Ozdemir et al. 2010).

In the most of the genera understudy uniseriate multicellular trichomes with the glandular cell at apex along with unicellular eglandular trichomes were found. Species of Silene, Shivparvatia, Stellaria, Cerastium, Odontostemma, Eremogone, Arenaria, Lepyrodiclis and Sagina studied during present study possess both uniseriate glandular and eglandular trichomes. Metcalfe \& Chalk (1950) mentioned presence of uniseriate multicellular trichomes with glandular cell at apex in the species of Dysphania (D. myriocephala Benth.), Habrosia, Silene, Spergula, Spergularia and Loeflingia. Al-Saadi \& Al-Taie (2014) reported presence of uniseriate multicellular glandular and eglandular trichomes in different Minuartia species. Some completely glabrous species Stellaria depressa, Spergula fallax and Cerastium davuricum reported during present work Corrigiola sp., and Telephium imperati L. also show similar pattern (https://www.infoflora.ch/fr/flore/339telephium-imperati.html, https://florabase.dpaw.wa.gov.au/browse/profile/22402).

In Caryophyllaceae, trichome presence and diversity can be used for delimitation of certain species i.e. Spergula fallax can be distinguished from morphologically similar Spergularia rubra due to having fully glabrous plant body. Drymaria villosa has strictly long multicellular uniseriate eglandular trichomes while its allied species D. cordata has unicellular glandular and eglandular trichomes. S. incurvifolia possess both multicellular uniseriate glandular and multicellular uniseriate eglandular trichomes while allied S. incurvifolia due to the presence of only multicellular uniseriate eglandular trichomes. Thus, Silene moorcroftiana can also be distinguished from former due to presence of the trichomes. Silene kumaonensis can also be distinguished from closely related $S$. indica by presence of only multicellular uniseriate eglandular trichomes, while both multicellular uniseriate glandular and multicellular uniseriate eglandular trichomes are found in S. indica. Shivparvatia ciliolata can be distinguished from $S$. glanduligera due to presence of only multicellular uniseriate eglandular trichomes, while $S$. glanduligera possess both multicellular uniseriate glandular and multicellular uniseriate eglandular trichomes. Eremogone kumaonensis and E. festucoides can also be distinguished by due to 
presence of trichomes. E. kumaonensis contains multicellular uniseriate eglandular hairs and in E. festucoides both multicellular uniseriate glandular and multicellular uniseriate eglandular hairs present. Sagina apetala and S. saginoides also show same pattern. In S. saginoides only multicellular uniseriate eglandular trichomes are found, while both multicellular uniseriate glandular and multicellular uniseriate eglandular trichomes are found in $S$. apetala. Vestiture type can also be used to differentiate closely allied Stellaria congestiflora and $S$. decumbens. Stellaria congestiflora has villous vestiture while $S$. decumbens has strigulose vestiture. Similarly, Silene indica var. indica and S. indica var. edgeworthii can also be distinguished due to presence of tomentose vestiture in first and strigulose vestiture in later. Closely allied Arenaria serpylifolia and A. neelgherrensis can be distinguished on the basis of hairy leaf surface and sepal in first and glabrous leaf surface and sepal in the second. Shivparvatia ciliolata var. ciliolata and Shivparvatia ciliolata var. pendula also differ due glabous leaf surface in the first variety and hairy leaf surface in second variety.

Trichome diversity and distribution in some angiosperm family as Asteraceae (Krak \& Mraz 2008, Angulo \& Dematteis 2014, de Andrade Wagner et al. 2014), Brassicaceae (Abdel 2005, Beilstein et al. 2006), Cucurbitaceae (Ali \& Al-Hemaid 2011) Ranunculaceae (Hoot 1991), and some genera as Colquhounia (Hu et al. 2012), Teucrium (Navarro \& El Oualidi 1999) of Lamiaceae has taxonomic significance and can be used for delimitation of tribes, genera, sections and series. But in the family Caryophyllaceae trichomes diversity and distribution does not have such significance.

After perusing literature and present work trichome types of 33 genera belonging to all eleven tribes of the family analysed and summarized in table 3. Interestingly, glabrous plants present in all tribes of the family. Further, it is concluded that trichome diversity and distribution does not play any significant role in the taxonomic delimitation neither generic nor tribal level of the family. Although, few closely allied species can be distinguished from each other either on the basis of presence of trichomes or vestiture pattern.

Table 3. Tribes and trichome types of the family Caryophyllaceae (after pursuing literature and present study).

\begin{tabular}{|c|c|c|c|}
\hline S.N. & Tribe & Genera studied & Trichomes \\
\hline 1. & Sclerantheae & Cherleria & $\begin{array}{l}\text { Uniseriate multicellular eglandular and } \\
\text { glandular trichomes }\end{array}$ \\
\hline 2. & Saginaea & Sagina and Habrosia & $\begin{array}{l}\text { Uniseriate multicellular eglandular and } \\
\text { glandular trichomes }\end{array}$ \\
\hline 3. & Spergulae & Spergula and Spergularia & $\begin{array}{l}\text { Uniseriate multicellular eglandular } \\
\text { trichomes }\end{array}$ \\
\hline \multirow[t]{5}{*}{4.} & Polycarpeae & $\begin{array}{l}\text { Drymaria, Polycarpaea, Polycarpon, } \\
\text { Loeflingia, Achyronichia, Stipulicida and } \\
\text { Krouseola }\end{array}$ & $\begin{array}{l}\text { Uniseriate multicellular glandular and } \\
\text { Eglandular }\end{array}$ \\
\hline & Exceptions & Drymaria cordata & Capitate glandular \\
\hline & & $\begin{array}{l}\text { Polycarpon prostratum, Polycarpaea sp. } \\
\text { and Stipulicida sp }\end{array}$ & Bifurcate eglandular \\
\hline & & Krouseola sp. and Achyronichia sp. & Eglandular multiseriate \\
\hline & & Polycarpon loeflingiae & Stellate \\
\hline \multirow[t]{3}{*}{5.} & Paronychieae & $\begin{array}{l}\text { Herniaria, Paronychia, Siphonychia and } \\
\text { Pollichia }\end{array}$ & Unicellular eglandular trichomes \\
\hline & Exception & Pollichia compestris & Bifurcate eglandular \\
\hline & & Herniaria cachemiriana & Multiseriate eglandular \\
\hline 6. & Corrigioleae & Corrigiola and Telephium & Glabrous \\
\hline \multirow[t]{3}{*}{7.} & Alsineae & $\begin{array}{l}\text { Cerastium, Lepyrodiclis, Odontostemma, } \\
\text { Shivpaarvatia and Stellaria }\end{array}$ & $\begin{array}{l}\text { uniseriate multicellular eglandular and } \\
\text { glandular trichomes }\end{array}$ \\
\hline & Exceptions & Stellaria vestita and Cerastium mollissimum & Stellate \\
\hline & & Cerastium dicrotrichum & eglandular bifurcate \\
\hline 8. & Arenarieae & Arenaria & $\begin{array}{l}\text { Uniseriate multicellular eglandular and } \\
\text { glandular trichomes }\end{array}$ \\
\hline 9. & Eremogoneae & Eremogone and Thylacospermum & $\begin{array}{l}\text { Uniseriate multicellular eglandular and } \\
\text { glandular trichomes }\end{array}$ \\
\hline \multirow[t]{2}{*}{10.} & Sileneae & Agrostemma, Silene and Viscaria & $\begin{array}{l}\text { Uniseriate multicellular eglandular and } \\
\text { glandular trichomes }\end{array}$ \\
\hline & Exceptions & Viscaria sp. and Silene propinqua & Capitates glandular trichome \\
\hline 11. & Caryophylleae & $\begin{array}{l}\text { Acanthophyllum, Dianthus, Gypsophila and } \\
\text { Vaccaria }\end{array}$ & $\begin{array}{l}\text { Uniseriate multicellular eglandular and } \\
\text { glandular trichomes }\end{array}$ \\
\hline
\end{tabular}




\section{ACKNOWLEDGEMENTS}

Authors are thankful to Herbarium curator of Botanical Survey of India Northern Circle Dehradun (BSD), Forest Research Institute Dehradun (DD), H.N.B. Garhwal University Srinagar Garhwal (GUH), Kumaon University Nainital (KUH) and Wildlife Institute of India Dehradun for providing access to their herbaria and libraries. Authors also extend thanks to the anonymous reviewers for their suggestions and critical comments.

\section{REFERENCES}

Abdel KK (2005) Morphological studies on trichomes of Brassicaceae in Egypt and taxonomic significance. Acta Botanica Croatica 64(1): 57-73.

Ali MA \& Al-Hemaid FM (2011) Taxonomic significance of trichomes micromorphology in Cucurbits. Saudi Journal of Biological Sciences 18(1): 87-92.

Al-Saadi SAMS \& Al-Taie S (2014) Taxonomic significance of anatomical characters in some species of Minuartia L. (Caryophyllaceae). Global Journal of Biology, Agriculture and Health Sciences 3(4):138-146.

Angulo MB \& Dematteis M (2014) Floral microcharacters in Lessingianthus (Vernonieae, Asteraceae) and their taxonomic implications. Plant Systematics and Evolution 300(8): 1925-1940.

Arcus MG, Lilios E, Doroftei \& Doicescu D (2012) The histo-anatomocal analysis of the species Cerastium tomentosum (Caryophyllaceae). Annals of RSCB 17(1): 350-354.

Bagci Y \& Bicer H (2015) Analysis of anatomical and morphological characters of the Silene cappadocica Boiss. \& Heldr. and Silene spergulifolia Bieb. (Caryophyllaceae) species. Scientific Papers. Series B, Horticulture 61: 293-302.

Beilstein MAIA, Al-Shehbaz \& Kellog EA (2006) Brassicaceae phylogeny and trichome evolution. American Journal of Botany 93(4): 607-619.

Bittrich V (1993 a) Introduction to Centrospermae. In: Kubitzki K, Rohwer JG \& Bittrich V (eds) The families and genera of vascular plants. Volume 2, Magnoliid, Hamamelid, and Caryophyllid families. Germany, Springer Science, pp. 13-19.

Bittrich V (1993 b) Introduction to Centrospermae. In: Kubitzki K, Rohwer JG \& Bittrich V (eds) The families and genera of vascular plants. Volume 2, Magnoliid, Hamamelid, and Caryophyllid families. Germany, Springer Science, pp. 206-236.

Bozchaloyi SE \& Keshavarz M (2014) Micro- and macromorphological study of Stellaria (Caryophyllaceae) and its closest relatives in Iran. Phytologia Balcanica 20(2): 179-197.

Cowie ID (1994) Three new species, a new name and notes on Australian Polycarpaea (Caryophyllaceae). Nuytsia 9(3): 319-332.

de Andrade Wagner MBFP, Loeuille CM, Siniscalchi GF, Melo-de-Pinna \& Pirani JR (2014) Diversity of nonglandular trichomes in subtribe Lychnophorinae (Asteraceae: Vernonieae) and taxonomic implications. Plant Systematics and Evolution 300(5): 1219-1233.

Dillenberger MS \& Kadereit JW (2014) Maximum polyphyly: Multiple origins and delimitation with plesiomorphic characters require a new circumscription of Minuartia (Caryophyllaceae). Taxon 63(1): 6488.

Greenberg AK \& Donoghue MJ (2011) Molecular systematics and character evolution in Caryophyllaceae. Taxon 60(6): 1637-1652.

Harbaugh DT, Nepokroeff M, Rabeler RK, McNeill J, Zimmer EA \& Wagner WL (2010) A new lineage-based tribal classification of the family Caryophyllaceae. The International Journal of Biological Sciences 171(2): 185-198.

Hoot SB (1991) Phylogeny of the Ranunculaceae based on epidermal microcharacters and macromorphology. Systematic Botany 16(4): 741-755.

Hu GX, Balangcod T, \& Xiang CL (2012) Trichome micromorphology of the Chinese-Himalayan genus Colquhounia (Lamiaceae), with emphasis on taxonomic implications. Biologia 67(5): 867-874.

Keshavarzi M \& Bozchaloyi SE (2014) Leaf and stem comparative anatomical analysis of three genera of Alsinoideae (Caryophyllaceae). Iranian Journal of Botany 20(1): 71-79.

Khan G, Zhang F, Gao Q, Mashwani Z, Rehman K, Khan MA \& Chen S (2013) Trichomes diversity in the tropical flora of Pakistan. Journal of Medicinal Plants Research 7(22): 1587-1592.

Kilic S (2009) Anatomical and pollen characters in the Genus Silene L. (Caryophyllaceae) from Turkey. Botany Research Journal 2(2-4): 34-44.

Krak K \& Mraz P (2008) Trichomes in the tribe Lactuceae (Asteraceae) - taxonomic implications. Biologia 63(5): 616- 
630.

Lawrence GHM (1951) Taxonomy of vascular plants. Macmillan, New York, 823 p.

Mabberley DJ (2017) Mabberley's plant-book: a portable dictionary of plants, their classifications, and uses. Cambridge, Cambridge University Press, 1102 p.

Majumdar NC (1993) Caryophyllaceae. In: Sharma BD \& Balakrishnan NP (eds) Flora of India. Volume 2. Calcutta, Botanical Survey of India. pp. 503-595.

Metcalfe CR \& Chalk L (1950) Caryophyllaceae. In: Metcalfe CR \& Chalk L (eds) Anatomy of the Dicotyledons. Volume 1. London, Oxford University Press, pp.147-152.

Navarro T \& Oualidi JEl (1999) Trichome morphology in Teucrium L. (Labiatae). A taxonomic review. Anales del Jardín Botánico de Madrid 57(2): 277-297.

Ozdemir C, Ozkan M \& Kandemir A (2010) The morphological and anatomical properties of Gypsophila lepidioides Boiss (Caryophyllaceae) endemic to Turkey. The International Research Journal of Plant Science 1(4): 69-74.

Payne WW (1978) A glossary of plant hair terminology. Brittonia 30(2): 239-255.

Prabhakar M \& Leelavathi P (1989) Structure, delimitation, nomenclature and classification of plant trichomes. Asian Journal of Plant Science (1): 49-66.

Pusalkar PK \& Singh DK (2015) Taxonomic rearrangement of Arenaria (Caryophyllaceae) in Indian Western Himalaya. Journal of Japanese Botany 90(2): 77-91.

Sadeghian S, Zarre S, Rabeler RK \& Heubl G (2015) Molecular phylogenetic analysis of Arenaria (Caryophyllaceae: tribe Arenarieae) and its allies inferred from nuclear DNA internal transcribed spacer and plastid DNA rps16 sequences. The Botanical Journal of the Linnean Society 178(4): 648-669.

Sahreen S, Khan MA, Khan MR \& Khan RA (2010) Leaf epidermal anatomy of the genus Silene (Caryophyllaceae) from Pakistan. Biological Diversity and Conservation 3(1): 93-102.

Selvi S, Guner O \& Akcicek E (2014) Anatomical, micromorphological and ecological studies on the genus Agrostemma L. (Caryophyllaceae) growing in Turkey. Biological Diversity and Conservation 7(1): 61-67.

Shamsabad MM, Ejtehadi H, Vaezi J \& Memariani F (2013) Anatomical and pollen characters in Acanthophyllum Ca Mey. (Caryophyllaceae) from northeast of Iran. The Iranian Journal of Botany 19(1): 107-118.

Simpson MG (2010) Plant Systematics, $2^{\text {nd }}$ edition. Amsterdam, Elsevier, $740 \mathrm{p}$.

Solereder H \& Scott DH (1986) Systematic anatomy of the dicotyledons: a handbook for laboratories of pure and applied botany. Volume 2. New Delhi. Ajay book service, $666 \mathrm{p}$.

The Plant List (2013) Version 1.1 Published on the Internet. Available from: http://www.theplantlist.org/ (accessed 22 June 2019).

Thorne RF \& Reveal JL (2007) An updated classification of class Magnoliopsida (“Angiosperm”). The Botanical Review 73: 67-182.

Werker E (2000) Trichome diversity and development. Advances in Botanical Research 31: 1-35.

Yildiz K \& Minareci E (2008) Morphological, anatomical, palynological and cytological investigation on Silene urvillei Schott. (Caryophyllaceae). Journal of Applied Biological Sciences 2(2): 41-46. 